\title{
Lucas-Washburn vs Richards equation for the modelling of water absorption in cementitious materials
}

\author{
Yury Villagrán Zaccardi ${ }^{1, *}$, Natalia Alderete ${ }^{1,2}$, and Nele De Belie ${ }^{2}$ \\ ${ }^{1}$ LEMIT, CONICET, 52 entre 121 y 122 s/n, 1900 La Plata, Argentina \\ ${ }^{2}$ Magnel Laboratory for Concrete Research, Department of Structural Engineering, Faculty of Engineering and Architecture, Ghent \\ University, Tech Lane Ghent Science Park, Campus A, Technologiepark Zwijnaarde 904, B-9052 Ghent, Belgium
}

\begin{abstract}
The Lucas-Washburn equation is still being applied by a significant number of researchers for the modelling of water absorption in cementitious materials. A modern approach considers the extended Darcy's law leading to the Richards equation instead. Three main assumptions are implied by the application of the Lucas-Washburn equation: the flow occurs in one direction only, the material is separated into one fully wet and one fully dry region, and pores are modelled as an assembly of parallel tubes of a particular radius. Its application to analyse experimental results allows defining these three assumptions as mere simplifications. Therefore, all the parameters comprised in the Lucas-Washburn model are apparent. Consequently, a very limited description of the transport properties of the material can be achieved. For many engineering purposes this would not be an issue, but for an intrinsic description of the material a more realistic model is required. This paper discusses the limitations of the Lucas-Washburn equation, and the advantages of the Richards equation regarding the modelling of water absorption in cementitious materials. The comparative analysis reveals the versatility of the Richards equation, with an approach that considers the material as a continuum and describes it through measurable parameters.
\end{abstract}

\section{Introduction}

Transport properties of concrete are defined by its pore size distribution and connectivity. Unsaturated concrete in direct contact with water takes it up by capillary absorption, due to the action of forces of adhesion of water molecules to the pore walls in concrete. The ingress of aggressive agents into concrete may be promoted by this mechanism affecting durability.

Capillary absorption rate is a parameter that can be used as a design tool for durable concrete. There are plenty of examples in the literature of relationships between the water sorptivity coefficient (WSC) or sorptivity (generally expressed in units of weight per square meter over the square root of time: mass/area/time ${ }^{0.5}$ ) and other durability and transport properties (drying rate [1], chloride diffusion [2], carbonation [3], water penetration under pressure [2, 45], resistance against freeze and thaw [6-8]). Consequently, the sorptivity is a qualitative descriptor of transport properties of concrete, with a low rate of water absorption indicating satisfactory properties to ensure an acceptable lifespan of a concrete structure. The WSC of concrete can be applied as an index for a performancebased approach for durability of reinforced concrete structures. However, it is still very limited as a performance-based design tool for durability as it is unable to provide reliable estimations of values for other transport properties of concrete.

The main limitation of current practice is the requirement of adopting conventional procedures for obtaining the value of the WSC from experimental results due to a lack of linearity with $t^{0.5}$. The WSC is generally computed as the slope of the fitting line to the amount of water uptake per unit area as a function of the square root of the time along which concrete was in contact with water. This approach is applied to analyse capillary absorption in most of porous materials and also in cementitious materials. However, there is a particular anomaly consistently reported in the literature showing a lack of linearity of the evolution of water uptake of cementitious materials with $t^{0.5}$. The analysis of the reasons for this lack of linearity and their inclusion in the transport model require to look into the fundamentals of the transport model. A detailed analysis and a potential explanation for this anomaly can be found in [9], where a better correlation with $t^{0.25}$ is sustained.

The insight of the phenomenon in the search of finding a full descriptive model requires the consideration of several variables for the case of cementitious materials. There are two possible approaches for the modelling of capillary absorption in porous materials. These are the Lucas-Washburn (LWE) and the Richards (RE) equations. The LWE was the first implemented for describing the process on the basis of

* Corresponding author: yuryvillagran@conicet.gov.ar 
hydrostatic condition of water in capillary pores. Alternatively, the RE is based on a balance of mass in the porous material.

\section{The Lucas-Washburn Equation}

The earliest solution for the relationship between water uptake by unsaturated porous materials and time is the LWE [10], which combines the Laplace relation with Poiseuille's equation of laminar flow. This indicates that the water front and mass versus time follow the relation of $t^{0.5}$ in a small cylindrical capillary.

When the gravitational and inertial terms are neglected, the capillary force is balanced by the viscous force. See Eq. (1), where $\sigma$ is the surface tension of the liquid, $\hat{h}$ is the rate of increase in the meniscus height inside the capillary, $h$ is the meniscus height inside the capillary, $\theta_{d y n}$ is the dynamic contact angle, $R_{c}$ is the capillary radius, $R_{h}$ is the hydraulic radius, $t$ is time, $\mu$ is the liquid viscosity. The solution of this equation can be obtained by considering the initial condition $h(t=0)=0$, and it is the well-known LWE, Eq. (2), where $R_{e}$ is obtained from Eq. (3).

$$
\begin{gathered}
\frac{2 \sigma \cdot \cos \left(\theta_{d y n}\right)}{R_{c}}=\frac{8 \mu h \hat{h}}{R_{h}^{2}} \\
h=\sqrt{\frac{\sigma \cdot R_{e} \cos \left(\theta_{d y n}\right)}{2 \mu} \cdot t}=k \sqrt{t} \\
R_{e}=\frac{R_{h}{ }^{2}}{R_{c}}
\end{gathered}
$$

As said, the LWE relates the height of the water front with the square root of time. In practice, the gravimetric method is applied, and considering 1-D transport, the height increase is revealed by the proportional weight increase.

The application of LWE to porous media requires to adopt a model of the material composed of a solid containing idealised capillary pores in the shape of parallel cylinders. This is certainly a significant simplification and several authors have made attempts to overcome the subsequent limitations.

The LWE has been studied, improved and applied very extensively, but it has several inherent weaknesses and problems when applied to model water uptake of porous materials [11]:

(1) The capillary model considers 1-D flow in straight tubes. It is therefore applicable to $1-\mathrm{D}$ wicking flow only.

(2) The assumption of a bundle of capillary tubes for representing the actual complex microstructure of a porous medium is very simplistic.

(3) There is no consideration of the interconnection of pores.

(4) The distribution of pore diameters cannot be assessed, and only a constant capillary tube diameter is assumed.
(5) The tortuosity is disregarded, and the actual path of the flow is replaced with a straight-line fluid motion.

All these assumptions have been considered in improved models, leading to the inclusion of respective fitting parameters, such as hydraulic diameter, capillary diameter, and tortuosity factors. Pores in cementitious materials are certainly not cylindrical and have no constant radius. Several attempts to adapt the LWE for considering the shape and tortuosity have been made [12]. However, these approaches require additional assumptions as they are based on a $t^{0.5}$ evolution. The lack of linearity documented for several materials is therefore considered evidence of the unsuitability of the LWE for the description of imbibition in 3-D porous materials [12].

The LWE is still extensively applied for the study of imbibition in porous materials, but its limitations result in an empirical approach when a full description of the phenomenon is attempted. The main reasons for its use seem to be its simplicity, small number of simple parameters to be measured, direct validation, and universality of the model. However, more complex approaches are possible nowadays in the search of a better understanding of the capillary imbibition of cementitious materials.

\section{The Richards Equation}

The RE is originated by the application of the so-called extended Darcy Equation (Eq. 4) for describing unsaturated flow of water in porous media $(\boldsymbol{u})$ [13]. Where $K(\theta)$ is the hydraulic conductivity (with $\theta$ the volume fraction saturation), and $\boldsymbol{F}$ is the capillary force, which is identified with the negative gradient of the capillary potential $\psi$. Combining Eq. 4 with the continuity equation leads to the Richards equation, fundamental equation of the unsaturated flow (Eq. 5). Defining the hydraulic diffusivity function, $D=$ $K(d \psi / d \theta)$, it becomes Eq. 6 in one dimension, with $x$ being the distance.

$$
\begin{gathered}
\boldsymbol{u}=K(\theta) \boldsymbol{F}=-K(\theta) \nabla \psi \\
\frac{\partial \theta}{\partial t}=\nabla K(\theta) \nabla \psi \\
\frac{\partial \theta}{\partial t}=\frac{\partial}{\partial x}\left(D \frac{\partial \theta}{\partial x}\right)
\end{gathered}
$$

For solving this equation, the Boltzmann transformation $\left(\phi=x \cdot t^{-0.5}\right)$ is applied by defining $\theta=$ $f(\phi)$. Then, Eq. 6 is written as Eq. 7. With boundary conditions $\theta=\theta_{s}$ at $\phi=0$ (saturated at the surface in contact with water) and $\theta=\theta_{d}$ as $\phi \rightarrow \infty$ (homogeneously unsaturated semi-infinite medium), the solution becomes Eq. 8.

$$
-\frac{\phi}{2} \frac{d \theta}{d \phi}=\frac{d}{d \phi} D \frac{d \theta}{d \phi}
$$




$$
x(\theta, t)=\phi(\theta) \cdot t^{0.5}
$$

This last equation indicates that the profile of liquid content versus distance advances with $t^{0.5}$ maintaining the constant shape $\phi(\theta)$ during water uptake. When $\phi$ is known, the cumulative absorption $i$ is given by Eq. 9 [14], which is the basis for the most common approach in the analysis of capillary absorption in cementitious materials.

$$
i=t^{0.5} \int_{\theta_{d}}^{\theta_{s}} \phi d \theta=S \cdot t^{0.5}
$$

However, experimental results from cementitious materials do not fit well with Eq. 9. This application faces the significant difficulty of the lack of linearity of the relationship between water uptake and $t^{0.5}[13,15-$ 22]. This difficulty can be easily overcome when a variable hydraulic diffusivity is considered.

Note that the model considers no pore shape or size, and the pore structure is only defined by the transport properties of the material being tested.

\section{Discussion}

The LWE and RE are derived for the same purpose but they are different in their nature. Whereas LWE is based on an extremely simplistic geometrical model of the pore structure, RE defines the material as a continuum and considers its average properties. The required assumptions of the first model make it less adaptable than the second one. This would not be an issue if additional considerations were not necessary. Complex pore structures require the inclusion of abstract coefficients for LWE, whereas the RE can be adapted in a more genuine manner.

For example, for considering the lack of linearity with $t^{0.5}$, a fictional correlation coefficient has to be included in LWE, with no physical meaning and the only purpose of improving the fitting to experimental data. Despite some publications have proposed a modified LWE for accounting for the non-linearity of the process, these attempts end up in a completely empirical application as a convenient value for the hydraulic radius needs to be selected with the sole aim of obtaining a good fitting to experimental data. Contrarily, a variable hydraulic diffusivity may be considered in RE, and depending on its variation, the exponential parameter for $t$ may adopt different values. The variation of the hydraulic diffusivity can be obtained independently. This is the approach followed in [9], where an improved model based on RE is proposed for describing water absorption in cementitious materials, with water uptake increasing with $t^{0.25}$.

The previous approach is based on the fact that the unsaturated flow of water in cementitious materials requires an engineering description that is well satisfied by the RE. The need of a non-linear diffusion equation is sustained by the dependency of the process on time and moisture content. Despite the significant efforts made for connecting capillary suction with pore size distribution of the cementitious materials through the LWE, the value of this linking is relative when a process evolving with time and moisture content is proven. Significant variation of the pore size distribution during unsaturated transport of water in cementitious materials is manifest. Therefore, the main reasons for applying the LWE should be dismissed.

Some modern approaches consider fractal theory for the consideration of the complex and multidimensional structure of cementitious materials [23-27]. It is therefore possible to include transport processes such as one dimensional diffusion for long term exposure, to assess the experimental evidence of further increase in moisture content even after the capillary suction ceased $[9,28]$. This fractal approach is practical only in association with the Darcy's law, the same that gives origin to the RE [29]. An equivalent consideration for LWE has been attempted in [30], but the outcome of this approach implies the derivation of a significant number of correlation factors that cannot be explained from a physical point of view. Moreover, the most successful modern 3-D models of cementitious materials consider it as a granular material rather than a solid. Those models have been partially validated for other transport properties with very good chances of being applied to capillary suction.

A significant advantage of RE over LWE is the ability to consider a dependence of the transport coefficient on the water content [28]. With a rigid model for the geometry of the pore structure this is very difficult to achieve with the LWE, as all parameters and their evolution with $t$ and moisture content need to be modelled. The RE only requires the consideration of the evolution of diffusivity.

Another issue is the consideration of ink-bottle pores. Results from mercury intrusion porosimetry tests are commonly interpreted as a reflection of the presence of a significant volume of ink-bottle pores. Results from mercury intrusion porosimetry are also analysed with the LWE, and cyclic measurements are many times indicated for cementitious materials to account for the volume of ink-bottle pores. However, cyclic testing is not possible for the case of water penetration. The presence of some pores remains undercover and the relationship between sorptivity and other transport properties cannot be explained in absence of this consideration. This lack of information is a significant drawback for the application of the capillary water uptake analysed with the LWE to the design for durability based on performance. A more realistic description is necessary and this leads to abandon the application of the LWE for interpreting capillary suction of water.

Moreover, the LWE requires to be fed with parameters that can be measured macroscopically but not at the scale of pore sizes. For example, the contact angle must be supposed at a fixed value, but it is almost certain that this parameter will vary with pore size as it depends on the chemistry of pore walls. Even an average value of the contact angle cannot be measured experimentally as the meniscus formed in the pore structure cannot be visualized. 


\section{Conclusions}

The capillary water uptake of cementitious materials has been investigated from the practical and phenomenological points of view for many decades. The Lucas-Washburn (LWE) and Richards (RE) equations are the two possible approaches for this purpose.

From a comparative analysis between both approaches, it is derived that the RE is more versatile than the LWE in the sense that it considers the material as a continuum and defines its properties in accordance. Conversely, the LWE is based on the modelling of the pore structure geometry, which leads to a non-realistic approach. The main drawback of the LWE is that it cannot naturally adapt itself to reflect a pore structure evolving with time and moisture content.

Modelling based on the LWE should be abandoned in benefit of the RE, which is fed with measurable parameters.

\section{References}

1. Y.A. Villagrán Zaccardi, N.M. Alderete, A. Píttori, Á.A. Di Maio, XIII Conf. Durab. Build. Mater. Components, 336-34 (2014)

2. Y.A. Villagrán Zaccardi, V.L. Taus, Á.A. Di Maio, in: Av. en Segur. y Durabilidad. Ciclo Vida de las Estructuras. Auscultación y Diagnóstico de Estructuras Deterioradas, 237-253 (2011)

3. P.A.M. Basheer, D.P. Rankin, G.I.B. Russell, 8th Int. Conf. Durab. Build. Mater. Components, 423-435 (1999)

4. P. Van den Heede, E. Gruyaert, N. De Belie, Cem. Concr. Compos. 32, 749-756 (2010)

5. G.C. Long, K.L. Ma, Y.J. Xie, Adv. Mater. Res. 163-167, 3311-3319 (2011)

6. C. Hazaree, K. Wang, H. Ceylan, K. Gopalakrishnan, J. Mater. Civ. Eng. 23, 1181-1191 (2011)

7. D.P. Bentz, M.A. Ehlen, C.F. Ferraris, E.J. Garboczi, 7th Int. Conf. Concr. Pavements, 181-193 (2001)

8. V.N. Patel, Sorptivity Testing to Assess Durability of Concrete (McGill University, 2009).

9. Y.A. Villagrán Zaccardi, N.M. Alderete, N. De Belie, Cem. Concr. Res. 100, 153-165 (2017).
10. E.W. Washburn, Phys. Rev. XVII, 273-283(1921)

11. R. Masoodi and K.M. Pillai, in: Wicking in Porous Materials Traditional and Modern Modeling Approaches, 31-54 (2013).

12. J. Cai, B. Yu, Transp Porous Med 89, 251-263 (2011)

13. C. Hall, W.D. Hoff, Water Transport in Brick, Stone and Concrete, (2nd ed., CRC Press, Boca Raton, USA, 2009)

14. J.R. Philip, Trans. Faraday Soc. 51, 885-892 (1955).

15. W.P.S. Dias, Mag. Concr. Res. 56, 537-543(2004)

16. L.J. Parrott, Mater. Struct. 25, 284-292 (1992)

17. Y.A. Villagrán-Zaccardi, C.J. Zega, M.E. Sosa, $V$ Congr. Int. AATH, 87-94 (2012)

18. J. Kaufmann, W. Studer, Mater. Struct. 28, 115-124 (1995)

19. C. Hall, T.K.M. Tse, Build. Environ. 21, 113-118 (1986)

20. C. Hall, M.H. Raymond Yau, Build. Environ. 22, 7782 (1987)

21. N.S. Martys, C.F. Ferraris, Cem. Concr. Res. 27, 747-760 (1997)

22. B. Van Belleghem, R. Montoya, J. Dewanckele, N. Van den Steen, I. De Graeve, J. Deconinck, N. De Belie, Constr. Build. Mater. 110, 154-162 (2016)

23. J. Cai, B. Yu, X. Hu, in: Wicking in Porous Materials Traditional and Modern Modeling Approaches, 263294 (2013).

24. S. Havlin, D. Ben-avraham, Adv. Phys. 51 187-292 (2002)

25. M. Appel, G. Fleischer, J. Kärger, F. Fujara, S. Siegel, Europhys. Lett. 34 483-487 (1996)

26. S. Roux, F. Plouraboué, J.-P. Hulin, Transp. Porous Media 32 97-116 (1998).

27. M. Küntz, P. Lavallée, J. Phys. D. Appl. Phys. 34 2547-2554 (2001)

28. C. Hall, Cem. Concr. Res. 37 378-385 (2007).

29. H. Sun, M.M. Meerschaert, Y. Zhang, J. Zhud, W. Chen, Adv. Water Resources 52 292-295 (2013).

30. H.T. Nguyen, Water and heat transfer in cement based materials (University of Tromsø, 2011). 